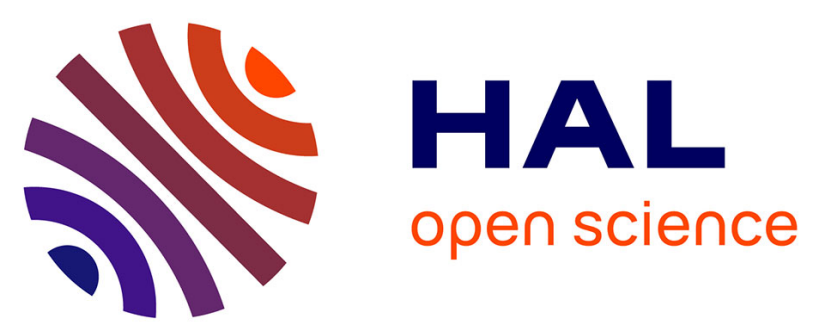

\title{
A New Computational Method for Prediction of Transient Hygroscopic Stresses during Moisture Desorption in Laminated Composite Plates with Different Degrees of Anisotropy
}

\author{
A. Tounsi, E. A. Adda Bedia, A. Benachour
}

\section{To cite this version:}

A. Tounsi, E. A. Adda Bedia, A. Benachour. A New Computational Method for Prediction of Transient Hygroscopic Stresses during Moisture Desorption in Laminated Composite Plates with Different Degrees of Anisotropy. Journal of Thermoplastic Composite Materials, 2005, 18 (1), pp.37-58. 10.1177/0892705705041156 . hal-00570792

\section{HAL Id: hal-00570792 \\ https://hal.science/hal-00570792}

Submitted on 1 Mar 2011

HAL is a multi-disciplinary open access archive for the deposit and dissemination of scientific research documents, whether they are published or not. The documents may come from teaching and research institutions in France or abroad, or from public or private research centers.
L'archive ouverte pluridisciplinaire HAL, est destinée au dépôt et à la diffusion de documents scientifiques de niveau recherche, publiés ou non, émanant des établissements d'enseignement et de recherche français ou étrangers, des laboratoires publics ou privés. 


\title{
A New Computational Method for Prediction of Transient Hygroscopic Stresses during Moisture Desorption in Laminated Composite Plates with Different Degrees of Anisotropy
}

\author{
A. Tounsi, ${ }^{*}$ E. A. Adda Bedia And A. Benachour \\ Laboratoire des Matériaux et Hydrologie \\ Université de Sidi Bel Abbes \\ BP 89 Cité Ben M'hidi 22000 Sidi Bel Abbes, Algérie
}

\begin{abstract}
A theoretical study is presented for predicting the hygrothermal behavior of laminated composite plates during moisture desorption. This investigation is aimed at overcoming the computational difficulties in the determination of transient hygroscopic stress distribution through laminated plates and in the evaluation of the effect of anisotropy on the evolution of such stresses. To calculate such stresses during desorption phase, we present a new method by which we are able to predict directly the evolution of transient hygroscopic stresses within laminated composite plates without the calculation of moisture concentrations as in the case of Benkeddad's method in which we are obliged to determine the moisture distribution across the plate before the calculation of such stresses. This is very important and must be taken into account in the design of composite material, particularly aerospace structures for example, aircraft.

The second part of this investigation is devoted to the analysis of the effect of anisotropy on the hygrothermal behavior of laminated plates during moisture desorption. The polar representation method of anisotropic properties is selected as a convenient tool for this study. By this method, the author is able to assess the anisotropy of composite laminated plates using the degree of anisotropy. Some stacking sequences with different in-plane degree of anisotropy were investigated in order to show the effect of anisotropy on the transverse hygroscopic stresses. Finally, the results of this paper can be used to guide the derivation of future by opening a way for the optimal design of composite structures submitted to a moist environment.
\end{abstract}

*Author to whom correspondence should be addressed. E-mail: touabdel@yahoo.fr 
KEY WORDS: laminated composite plates, moisture desorption, transient hygroscopic stresses, polar representation method, degree of anisotropy.

\section{INTRODUCTION}

$\mathbf{I}$

N RECENT YEARS, fiber-reinforced composite laminated structures have been widely used in the aerospace, marine, automobile and other engineering industries. During the operational life, these laminates are subjected to complex environmental conditions in terms of temperature and moisture. The transient and nonuniform moisture concentration distributions in laminated composite plates give rise to transient and nonuniform stress fields $[1-3,16]$ which are investigated here. In order to design structure properly it is necessary to understand the hygrothermal behavior of such structures.

In the present study, the ambient temperature is assumed to have a uniform distribution, but the moisture is supposed to have a transient and nonuniform distribution. These assumptions are justified in this case for the same reasons (polymer matrix composite, thin plates) quoted [17-22]. As a result, the stress components due to a change of temperature can reasonably be computed using the classical laminated plate theory (CLPT) [23] due to the constant distribution of temperature across the plate. On the other hand, particular attention must be paid to the calculation of the transient stress components due to the nonuniform moisture concentration. It seems that there are few studies concerning transient hygroscopic stresses in laminated plates due to the nonuniform moisture distribution. Hahn and Kim [16] have discussed the distribution of such stresses in three particular cases of stacking sequences; Benkeddad et al. [2,3] have developed a numerical procedure to compute the in-plane hygroscopic stress components within the plies of laminated composite plates. More recently, Tounsi et al. [1] have developed a new analytical method to predict these stresses through the thickness of composite plates without knowing the moisture distribution during absorption. As a result, the rigorous distribution of hygroscopic residual stresses remains unknown in many cases.

This article consists of two parts. The first part deals with the calculation of the transient hygroscopic stresses during moisture desorption. For this, a new analytical method is developed while following the same methodology described in [1] with some modifications taking into account the phenomenon of desorption. The developed method, allows us to predict directly the transient hygroscopic stresses without the calculation of moisture concentration. 
In the second part of this article, we present a theoretical study concerning the effect of anisotropy on the variation of transient hygroscopic stresses in fiber-reinforced composite materials during moisture desorption. Evidently, the presence of a reinforcement in an isotropic matrix results in a general anisotropy for laminas; laminates obtained by superposing laminas with different orientations are then, in general, anisotropic as well, and their analysis by the CLPT needs the transformation of elastic properties of laminas by rotation. This transformation, though well known, is rather cumbersome, because it involves the fourth powers of trigonometric functions. Naturally, this condition is an obstacle to analytical manipulations, similar to those which occur in treating inverse problems for laminates; again, the complication of formulas can hide some mechanical properties and makes the quantification of anisotropy difficult.

To overcome all this, the polar representation method of plane elasticity tensors can be used. This method was introduced by Verchery as early as 1979 [24], and successively it has been developed by other authors (Grédiac et al. [25], Kandil et al. [26], Vannucci et al. [11,13], Vannucci et al. [14], Verchery [12], Vincenti et al. [10,15], Tounsi et al. [4-6], Verchery et al. [27]). It is very similar to that presented by Jones [28] and Grédiac [29]. In the theoretical framework of plane stress state, which is at the basis of CLPT, the polar method can be, used; its main advantage consists in

the fact that material and frame rotations are easily expressed, giving the scientist much simpler equations than those obtained by Cartesian transformations.

Another important feature of the method is the physical meaning of tensor polar components: in the case, for instance, of the elastic tensor, these are invariant parameters directly representing the symmetries of a given material. So, by polar components, it is possible to distinguish at a glance the kind of anisotropy of a lamina, independently on the reference frame where its elastic moduli are known. Also, using this method, it is possible to quantify the anisotropy of laminated composite plate by introducing a degree of anisotropy as it was shown by Cheikh Saad Bouh et al. [8,9] and Tounsi et al. [4-6].

\section{ANALYSIS}

Let us consider a laminated plate of thickness $h$ made of polymer matrix composite, submitted on its two sides to the same dry environment. The plate is considered to be infinite in both the $x$ and $y$ directions and the moisture content vary only in the $z$ direction. The problem is therefore one-dimensional. The initial moisture concentration $c_{\text {ini }}$ is uniform at $t=0$. Both sides of the plate are suddenly exposed to a zero moist environment 
in both faces of the plate. The moisture concentration inside the plate is described by the Fick equation with diffusivity $D$,

$$
\frac{\partial c(z, t)}{\partial t}=D \frac{\partial^{2} c(z, t)}{\partial z^{2}}
$$

with

$$
\begin{gathered}
C=C_{\text {ini }} \quad \text { at } t=0 \text { for } \frac{-h}{2}<z<\frac{h}{2} \\
C=0 \quad \text { at } t>0 \text { for } z=\frac{-h}{2} \text { and } z=\frac{h}{2}
\end{gathered}
$$

The residual stresses are computed using the following procedure, which is an extension of the method given by Tsai [23] for uniform moisture concentration distribution and of the method given by Benkeddad et al. [2,3] for linear moisture concentration distribution within the subply. The principle of this simplified method is to take the real distribution of moisture concentration through each ply using its serial expansion [30] and to determine the exact expression of the nonmechanical in-plane stress components $N_{\alpha}$ and moment components $M_{\alpha}$ (Equations (16) and (17)) without the term $C\left(z_{k}, t\right)$ representing the concentration of moisture.

Time $t$ being given, the first step is to compute the on-axis free expansions $e_{x}$ and $e_{y}$. These expansions are computed at each point $z_{k}$ of the thickness by the following equation:

$$
\begin{aligned}
& e_{x}^{(K)}=\alpha_{x} \Delta T+\beta_{x} \lambda_{k} \\
& e_{y}^{(K)}=\alpha_{y} \Delta T+\beta_{y} \lambda_{k} \\
& e_{S}^{(K)}=0
\end{aligned}
$$

with

$$
\begin{aligned}
C\left(z_{k}, t\right) & =\lambda_{k} \\
& =\left[\frac{4 c_{\text {ini }}}{\pi} \sum_{n=0}^{\infty} \frac{(-1)^{n}}{(2 n+1)} \cos \left(\frac{(2 n+1) \pi z_{k}}{h}\right) \exp \left(\frac{-D(2 n+1)^{2} \pi^{2} t}{h^{2}}\right)\right]
\end{aligned}
$$


The nonmechanical stresses $\sigma_{i}^{n(k)}, i=x, y$ are then easily computed in the ply frame

$$
\left\{\begin{array}{l}
\sigma_{x}^{n(k)}=Q_{x x} e_{x}^{(k)}+Q_{x y} e_{y}^{(k)} \\
\sigma_{y}^{n(k)}=Q_{y x} e_{x}^{(k)}+Q_{y y} e_{y}^{(k)}
\end{array}\right.
$$

In the laminate frame, these stresses become $\sigma_{\alpha}^{n(k)}, \alpha=1,2,6,[23]$

$$
\left\{\begin{array}{l}
\sigma_{1}^{n(k)}=p_{n}^{(k)}+q_{n}^{(k)} \cos 2 \theta \\
\sigma_{2}^{n(k)}=p_{n}^{(k)}-q_{n}^{(k)} \cos 2 \theta \\
\sigma_{6}^{n(k)}=q_{n}^{(k)} \sin 2 \theta
\end{array}\right.
$$

where

$$
\left\{\begin{array}{l}
p_{n}^{(k)}=\frac{Q_{x x}+Q_{x y}}{2} e_{x}^{(k)}+\frac{Q_{x y}+Q_{y y}}{2} e_{y}^{(k)} \\
q_{n}^{(k)}=\frac{Q_{x x}-Q_{x y}}{2} e_{x}^{(k)}+\frac{Q_{x y}-Q_{y y}}{2} e_{y}^{(k)}
\end{array}\right.
$$

Let $p_{\alpha n}, Q_{\alpha n}, p_{\beta n}$ and $Q_{\beta n}$ be the quantities defined by the relations:

$$
\begin{aligned}
& \left\{\begin{array}{l}
p_{\alpha n}=\frac{Q_{x x}+Q_{x y}}{2} \alpha_{x}+\frac{Q_{x y}+Q_{y y}}{2} \alpha_{y} \\
Q_{\alpha n}=\frac{Q_{x x}-Q_{x y}}{2} \alpha_{x}+\frac{Q_{x y}-Q_{y y}}{2} \alpha_{y}
\end{array}\right. \\
& \left\{\begin{array}{l}
p_{\beta n}=\frac{Q_{x x}+Q_{x y}}{2} \beta_{x}+\frac{Q_{x y}+Q_{y y}}{2} \beta_{y} \\
Q_{\beta n}=\frac{Q_{x x}-Q_{x y}}{2} \beta_{x}+\frac{Q_{x y}-Q_{y y}}{2} \beta_{y}
\end{array}\right.
\end{aligned}
$$

Relations (8) become

$$
\left\{\begin{array}{l}
p_{n}^{(k)}=p_{\alpha n} \Delta T+p_{\beta n} \lambda_{k} \\
q_{n}^{(k)}=Q_{\alpha n} \Delta T+Q_{\beta n} \lambda_{k}
\end{array}\right.
$$


Relations (7) become

$$
\left\{\begin{array}{l}
\sigma_{1}^{n(k)}=\left(p_{\alpha n}+Q_{\alpha n} \cos 2 \theta\right) \Delta T+\left(p_{\beta n}+Q_{\beta n} \cos 2 \theta\right) \lambda_{k} \\
\sigma_{2}^{n(k)}=\left(p_{\alpha n}-Q_{\alpha n} \cos 2 \theta\right) \Delta T+\left(p_{\beta n}-Q_{\beta n} \cos 2 \theta\right) \lambda_{k} \\
\sigma_{6}^{n(k)}=\left(Q_{\alpha n} \sin 2 \theta\right) \Delta T+\left(Q_{\beta n} \sin 2 \theta\right) \lambda_{k}
\end{array}\right.
$$

If we suppose that $\Delta T$ is constant in the thickness of laminate (As it was explained in the introduction) Relations (12) become

$$
\left\{\begin{array}{c}
\sigma_{1}^{n(k)}=A_{\theta}+B_{\theta} \lambda_{k} \\
\sigma_{2}^{n(k)}=C_{\theta}+D_{\theta} \lambda_{k} \\
\sigma_{6}^{n(k)}=E_{\theta}+F_{\theta} \lambda_{k}
\end{array}\right.
$$

with

$$
\left\{\begin{array}{l}
A_{\theta}=\left(p_{\alpha n}+Q_{\alpha n} \cos 2 \theta\right) \Delta T \\
B_{\theta}=p_{\beta n}+Q_{\beta n} \cos 2 \theta \\
C_{\theta}=\left(p_{\alpha n}-Q_{\alpha n} \cos 2 \theta\right) \Delta T \\
D_{\theta}=p_{\beta n}-Q_{\beta n} \cos 2 \theta \\
E_{\theta}=\left(Q_{\alpha n} \sin 2 \theta\right) \Delta T \\
F_{\theta}=Q_{\beta n} \sin 2 \theta
\end{array}\right.
$$

The nonmechanical in-plane stress components $N_{\alpha}^{n}$ and moment components $M_{\alpha}^{n}$ are

$$
N_{\alpha}^{n}=\int_{-h / 2}^{h / 2} \sigma_{\alpha}^{n} d z \quad \text { and } \quad M_{\alpha}^{n}=\int_{-h / 2}^{h / 2} z \sigma_{\alpha}^{n} d z \quad \alpha=1,2,6
$$

$N_{\alpha}^{n}$ and $M_{\alpha}^{n}$ are computed using the real distribution of the nonmechanical stresses $\sigma_{\alpha}^{n(k)}$ between the points $z_{k}$ and $z_{k+1}, k=0, \ldots, N-1$

$$
\left\{\begin{array}{l}
N_{1}^{n}=\sum_{k=0}^{N-1}\left[h_{k} A_{\theta}+\frac{4 c_{\text {ini }} B_{\theta}}{\pi} \sum_{n=0}^{\infty} \frac{(-1)^{n} h \psi_{k}}{(2 n+1)^{2} \pi} \exp \left(\frac{-D(2 n+1)^{2} \pi^{2} t}{h^{2}}\right)\right] \\
N_{2}^{n}=\sum_{k=0}^{N-1}\left[h_{k} C_{\theta}+\frac{4 c_{\text {ini }} D_{\theta}}{\pi} \sum_{n=0}^{\infty} \frac{(-1)^{n} h \psi_{k}}{(2 n+1)^{2} \pi} \exp \left(\frac{-D(2 n+1)^{2} \pi^{2} t}{h^{2}}\right)\right] \\
N_{6}^{n}=\sum_{k=0}^{N-1}\left[h_{k} E_{\theta}+\frac{4 c_{\text {ini }} F_{\theta}}{\pi} \sum_{n=0}^{\infty} \frac{(-1)^{n} h \psi_{k}}{(2 n+1)^{2} \pi} \exp \left(\frac{-D(2 n+1)^{2} \pi^{2} t}{h^{2}}\right)\right]
\end{array}\right.
$$




$$
\left\{\begin{array}{l}
M_{1}^{n}=\sum_{k=0}^{N-1}\left[\rho_{k} A_{\theta}+\frac{4 c_{\text {ini }} B_{\theta}}{\pi} \sum_{n=0}^{\infty} \frac{(-1)^{n} h \xi_{k}}{(2 n+1)^{2} \pi} \exp \left(\frac{-D(2 n+1)^{2} \pi^{2} t}{h^{2}}\right)\right] \\
M_{2}^{n}=\sum_{k=0}^{N-1}\left[\rho_{k} C_{\theta}+\frac{4 c_{\text {inin }} D_{\theta}}{\pi} \sum_{n=0}^{\infty} \frac{(-1)^{n} h \xi_{k}}{(2 n+1)^{2} \pi} \exp \left(\frac{-D(2 n+1)^{2} \pi^{2} t}{h^{2}}\right)\right] \\
M_{6}^{n}=\sum_{K=0}^{N-1}\left[\rho_{k} E_{\theta}+\frac{4 c_{\text {ini }} F_{\theta}}{\pi} \sum_{n=0}^{\infty} \frac{(-1)^{n} h \xi_{k}}{(2 n+1)^{2} \pi} \exp \left(\frac{-D(2 n+1)^{2} \pi^{2} t}{h^{2}}\right)\right]
\end{array}\right.
$$

with

$$
\begin{aligned}
h_{k}= & z_{k+1}-z_{k} \\
\rho_{k}= & \left(z_{k+1}^{2}-z_{k}^{2}\right) / 2 \\
\psi_{k}= & \left(\sin \left(\frac{(2 n+1) \pi z_{k+1}}{h}\right)-\sin \left(\frac{(2 n+1) \pi z_{k}}{h}\right)\right) \\
\xi_{k}= & {\left[z_{k+1} \sin \left(\frac{(2 n+1) \pi z_{k+1}}{h}\right)-z_{k} \sin \left(\frac{(2 n+1) \pi z_{k}}{h}\right)\right.} \\
& \left.+\frac{h}{(2 n+1) \pi}\left(\cos \left(\frac{(2 n+1) \pi z_{k+1}}{h}\right)-\cos \left(\frac{(2 n+1) \pi z_{k}}{h}\right)\right)\right]
\end{aligned}
$$

The nonmechanical strains are deduced using the usual strain-stress relation in laminated plates [23].

$$
\begin{aligned}
\left\{\varepsilon^{0 n}\right\} & =[\alpha]\left\{N^{n}\right\}+[\beta]\left\{M^{n}\right\} \\
\left\{k^{n}\right\} & =[\beta]^{t}\left\{N^{n}\right\}+[\delta]\left\{M^{n}\right\}
\end{aligned}
$$

The nonmechanical strains $\varepsilon_{\alpha}^{n(k)}$ are then computed at each point $z_{k}$ :

$$
\varepsilon_{\alpha}^{n(k)}=\varepsilon_{\alpha}^{0 n}+z_{k} k_{\alpha}^{n}
$$

In the ply frame, these nonmechanical strains become $\varepsilon_{i}^{n(k)}$. The residual strains are then defined by the difference between the nonmechanical strains and the free expansions:

$$
\varepsilon_{i}^{r(k)}=\varepsilon_{i}^{n(k)}-e_{i}^{(k)}
$$

The residual stresses $\sigma_{i}^{n(k)}$ in the ply frame are finally

$$
\sigma_{i}^{r(k)}=Q_{i j} \varepsilon_{j}^{r(k)}
$$


Each stress $\sigma_{i}^{n(k)}$ can be expressed by summation of two stresses which are originally different:

$$
\sigma_{i}^{r(k)}=\sigma_{\mathrm{ig}}^{r(k)}+\sigma_{\mathrm{it}}^{r(k)}
$$

$\sigma_{\text {ig }}^{r(k)}$ is the transient hygroscopic stress calculated with a null distribution of temperature within the plate $(\Delta T=0)$.

$\sigma_{\mathrm{it}}^{r(k)}$ is the thermal residual stress calculated with a null distribution of moisture concentration within the plate $\left(C\left(z_{K}, t\right)=0\right)$.

\section{POLAR REPRESENTATION METHOD AND THE COMPUTATION OF THE DEGREE OF ANISOTROPY}

We recall, ([24]) that in-plane elasticity, the three Cartesian components of symmetric second-order tensor $\mathbf{L}$ can be expressed by three other quantities, a scalar $T$, a modulus $R$ and an angle $\Phi$ :

$$
\begin{aligned}
& L_{11}=T+R \cos 2 \phi, \\
& L_{22}=T-R \cos 2 \phi, \\
& L_{12}=R \sin 2 \phi
\end{aligned}
$$

The reverse equations of Equation (23) can be expressed in complex form:

$$
\begin{aligned}
T & =\frac{L_{11}+L_{22}}{2}, \\
2 \mathrm{Re}^{2 i \phi} & =L_{11}-L_{22}+2 i \mathrm{~L}_{12} .
\end{aligned}
$$

$T, R$ and $\Phi$ are the polar components of $\mathbf{L}$; indeed, formulas (23) and (24) are the algebraic transposition of Mohr's circle geometric construction.

For the case of a fourth rank $\mathbf{L}$ having the typical symmetries of elasticity, its six Cartesian components are a function of six other parameters, $T_{0}, T_{1}$, $R_{0}, R_{1}, \Phi_{0}$ and $\Phi_{1}$ :

$$
\begin{aligned}
& L_{1111}=T_{0}+2 T_{1}+R_{0} \cos 4 \phi_{0}+4 R_{1} \cos 2 \phi_{1}, \\
& L_{1122}=-T_{0}+2 T_{1}-R_{0} \cos 4 \phi_{0}, \\
& L_{2222}=T_{0}+2 T_{1}+R_{0} \cos 4 \phi_{0}-4 R_{1} \cos 2 \phi_{1}, \\
& L_{1212}=T_{0}-R_{0} \cos 4 \phi_{0}, \\
& L_{1112}=R_{0} \sin 4 \phi_{0}+2 R_{1} \sin 2 \phi_{1}, \\
& L_{2212}=-R_{0} \sin 4 \phi_{0}+2 R_{1} \sin 2 \phi_{1} .
\end{aligned}
$$


The reverse equations of Equation (25) are, in complex form,

$$
\begin{aligned}
8 T_{0} & =L_{1111}+L_{2222}-2 L_{1122}+4 L_{1212}, \\
8 T_{1} & =L_{1111}+L_{2222}+2 L_{1122}, \\
8 R_{0} e^{4 i \phi_{0}} & =L_{1111}+L_{2222}-2 L_{1122}-4 L_{1212}+4 i\left(L_{1112}-L_{2212}\right), \\
8 R_{1} e^{2 i \phi_{1}} & =L_{1111}-L_{2222}+2 i\left(L_{1112}+L_{2212}\right) .
\end{aligned}
$$

Parameters $T_{0}, T_{1}$ are scalar, $R_{0}$ and $R_{1}$ moduli, $\Phi_{0}$ and $\Phi_{1}$ polar angles. The most important feature of polar components, is that for a rotation $\theta$ of the reference frame, $T, R, T_{0}, T_{1}, R_{0}, R_{1}$ and the difference $\Phi_{0}-\Phi_{1}$ are invariant, while the polar angles $\Phi, \Phi_{0}$ and $\Phi_{1}$ are simply changed into $\Phi-\theta, \Phi_{0}-\theta$ and $\Phi_{1}-\theta$.

The special application of this method is the definition of the degree of anisotropy $(\varepsilon)$ of a material. It is defined as the relative deviation in stiffness between the material and its isotropic part (the isotropic part of a material should be defined from polar components, but for a laminate, it can be more simply seen as the homogeneous material with properties identical to the inplane properties of a quasi-isotropic laminate). The degree of anisotropy so defined is positive and always less than 1 and it is given (the degree of anisotropy) by the following expression:

$$
\varepsilon=\sqrt{\frac{\left|R_{0} \mathrm{e}^{4 i \phi_{0}}\right|^{2}+4\left|R_{1} \mathrm{e}^{2 i \phi_{1}}\right|^{2}}{T_{0}^{2}+2 T_{1}^{2}+\left|R_{0} \mathrm{e}^{4 i \phi_{0}}\right|^{2}+4\left|R_{1} \mathrm{e}^{2 i \phi_{1}}\right|^{2}}}
$$

\section{MATERIAL USED FOR THE CALCULATION}

The material used for the simulations is a carbon/epoxy composite whose properties are given in Tables 1-3. For simplicity's sake, the calculations are made under the assumption that the elastic properties remain constant for the entire range of the moisture concentration; even if it has been observed in practice that these properties are slightly different for wet and dry conditions $[17,31]$.

\section{VALIDATION OF THE METHOD}

For the validation of the present method, we have checked the results obtained using the method developed by Benkeddad et al. [2,3]. To this end, we have used the same material and the same environmental conditions to 
Table 1. Mechanical properties of the material [23].

\begin{tabular}{lcccccccc}
\hline Material & $\begin{array}{c}E_{x} \\
(\mathrm{GPa})\end{array}$ & $\begin{array}{c}E_{y} \\
(\mathrm{GPa})\end{array}$ & $v_{x}$ & $\begin{array}{c}\mathbf{G}_{x y} \\
(\mathrm{GPa})\end{array}$ & $\begin{array}{c}\alpha_{x} \\
\left(\mathbf{1 0 ^ { - 6 }} \mathbf{K}^{-1}\right)\end{array}$ & $\begin{array}{c}\alpha_{y} \\
\left(\mathbf{1 0}^{-6} \mathbf{K}^{-1}\right)\end{array}$ & $\boldsymbol{\beta}_{x}$ & $\boldsymbol{\beta}_{y}$ \\
\hline Carbon/epoxy & 181 & 10.3 & 0.28 & 7.17 & 0.02 & 22.5 & 0 & 0.6 \\
\hline
\end{tabular}

Table 2. Environmental conditions and diffusion parameters [17].

\begin{tabular}{lcccc}
\hline Material & $\begin{array}{c}\text { Temperature } \\
\left({ }^{\circ} \mathbf{C}\right)\end{array}$ & $\begin{array}{c}\text { Relative } \\
\text { humidity (\%) }\end{array}$ & $\begin{array}{c}\mathbf{D} \\
\left(\mathbf{m m}^{2} / \mathbf{s}\right)\end{array}$ & $\begin{array}{c}\mathbf{C}_{\max } \\
(\%)\end{array}$ \\
\hline Carbon/epoxy & 20 & 100 & $2.27 \times 10^{-8}$ & 1.5 \\
\hline
\end{tabular}

Table 3. Strength data of the material used for simulations [23].

\begin{tabular}{lccccc}
\hline Material & $X(\mathrm{GPa})$ & $X^{\prime}(\mathrm{GPa})$ & $Y_{(\mathrm{GPa})}$ & $\boldsymbol{Y}^{\prime}(\mathrm{GPa})$ & $\boldsymbol{S}(\mathrm{GPa})$ \\
\hline Carbon/epoxy & 1500 & 1500 & 40 & 246 & 68 \\
\hline
\end{tabular}

those used by Benkeddad et al. [2,3]. The results plotted in Figures 2 and 7 which are obtained using the present method are in good agreement with those presented in $[2,3]$ for the case of moisture desorption.

\section{RESULTS AND DISCUSSION}

The study, here, has been focussed on the influence of anisotropy and heterogeneity on the transient residual stress fields throughout the thickness. The calculations of such stresses are carried out by the method presented below.

\section{Transient Hygroscopic Stresses in a Unidirectional Laminate}

The results below are given under the following assumptions. The temperature $\Delta T$ between the room temperature and the stress-free temperature is $\Delta T=-100^{\circ} \mathrm{C}$. The initial concentration $\left(C_{\text {ini }}\right)$ within the laminate is assumed to be equal to $C_{\max }$, given in Table 2. The plate is suddenly exposed to a moist environment characterized by a zero moisture concentration. Due to the symmetries of the present problem, the shear stress $\sigma_{S}^{r}$ is always zero in the ply frame. The two last stresses $\sigma_{x}^{r}$ and $\sigma_{y}^{r}$ are plotted in Figures 1 and 2. Four curves are particularly emphasized in all the figures as well as the following ones: the initial stress distribution $(t=0)$, 


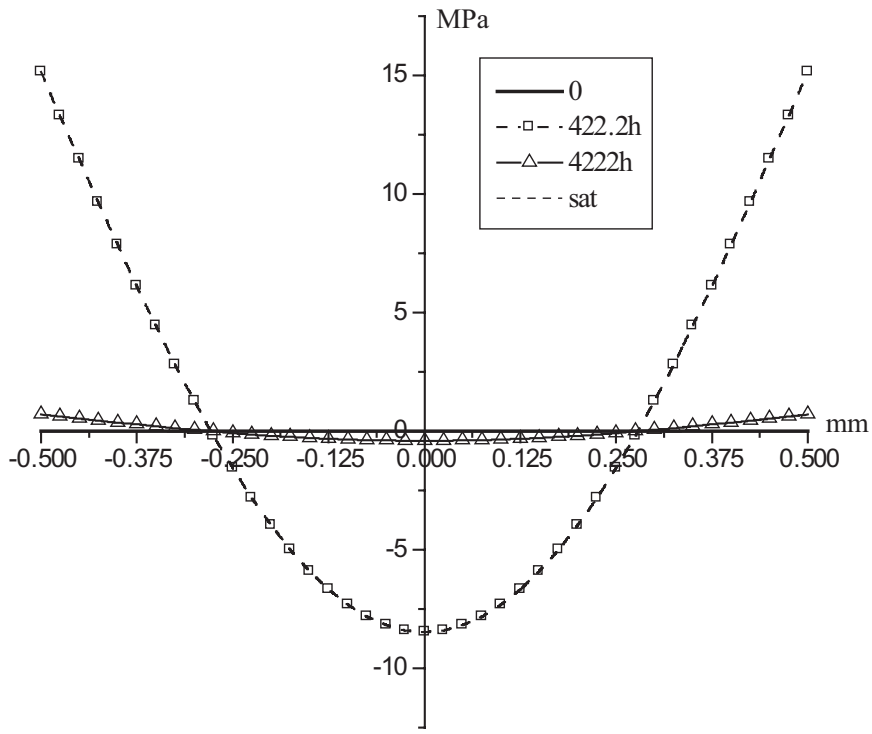

Figure 1. Residual longitudinal stress $\sigma_{x}^{r}$ through the thickness of a graphite/epoxy unidirectional laminate during desorption.

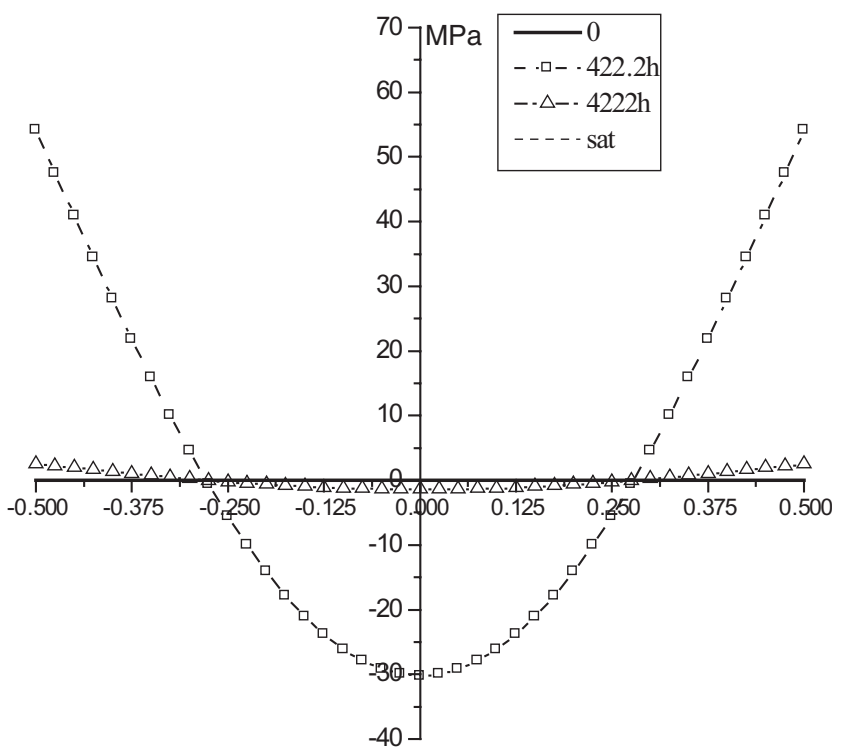

Figure 2. Residual transverse stress $\sigma_{y}^{r}$ through the thickness of a graphite/epoxy unidirectional laminate during desorption. 
the stress distribution when the moisture saturation is achieved (sat), stress distribution at $t=422.2 \mathrm{~h}$ and $t=4222 \mathrm{~h}$. In the present case, the two first distributions are zero. Both stresses $\sigma_{x}^{r}$ and $\sigma_{y}^{r}$ are compressive ones for the internal plies and tensile ones for the external plies. It can also be noted that the unstressed points remain the same from 422.2 to $4222 \mathrm{~h}$. They are approximately located at about one quarter of the thickness from both faces of the plate. The order of magnitude of $\sigma_{x}^{r}$ is negligible compared to both longitudinal tensile and compressive strengths $X$ and $X^{\prime}$ given in Table 3. On the other hand, the order of magnitude of the transverse stress $\sigma_{y}^{r}$ is important compared to both the transverse tensile and compressive strengths $Y$ and $Y^{\prime}$. The maximum value of the transverse stress $\sigma_{y}^{r}$ reaches $54.13 \mathrm{MPa}$ in the external plies at $422.2 \mathrm{~h}$. This value exceeds the transverse tensile strength $Y$, and the failure should theoretically occur. However, it must be pointed out that this calculation does not take into account the variation of elasticity modulus due to the humidity, which is likely to cause a stress relaxation.

\section{Transient Hygroscopic Stresses in Angle-ply $[+\theta,-\theta]_{25}$ Laminates}

The following angle-ply $[+\theta,-\theta]_{2 \mathrm{~S}}$ laminates have been studied: $\theta=10$, 20,30 and $45^{\circ}$. In all cases, it has been observed that the longitudinal stress $\sigma_{x}^{r}$ is negligible compared to longitudinal strengths $X$ and $X^{\prime}$. Apart from the case $\theta=45^{\circ}$, the shear stress $\sigma_{S}^{r}$ is not zero. A typical curve is depicted in Figure 3. The maximum value of the shear stress reaches $26.49 \mathrm{MPa}$ for $\theta=20^{\circ}$, i.e. about $38.95 \%$ of the shear strength $S$ given in Table 3. It can also be seen that the shear stress remains constant within each ply for any value of the time. This property has been observed for all laminates which have been presently studied. This is due to the definition of the residual strains which are the difference between the non-mechanical strains and the free expansions [Equation (20)]. The non-mechanical strains are constant within each plies for symmetric boundary conditions and uncoupled laminates. The free shear expansion is zero [Equation (4)]. As a result, the residual shear strains and the residual shear stress are constant within the plies. This property is also remarked in the case of moisture absorption $[1,4,5]$.

The transverse stress $\sigma_{y}^{r}$ is plotted in Figures 4-7 for the different values of $\theta$. The special case $\theta=0$ is plotted in Figure 2. First, the curves are approximately continued from a ply to another. As for the unidirectional laminate, the external plies are subjected to a tensile transverse stress during the moisture desorption except when $t=0$, where the plies are in compressive state. It is clearly shown in Figure 2 that transverse tensile and compressive stress peaks occur in the external and central plies, 


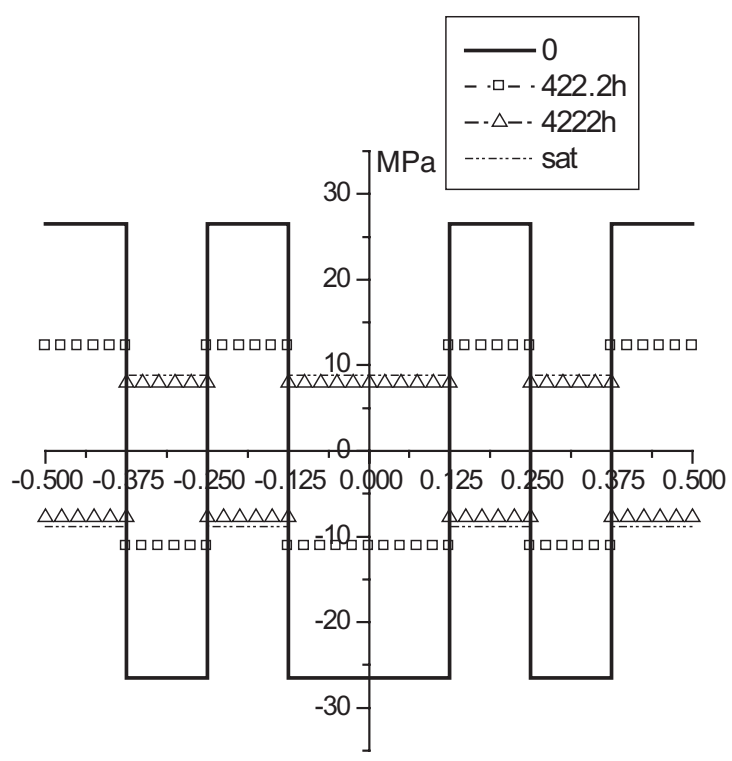

Figure 3. Residual shear stress $\sigma_{s}^{r}$ through the thickness of a graphite/epoxy $[+20,-20]_{2 S}$ during desorption.

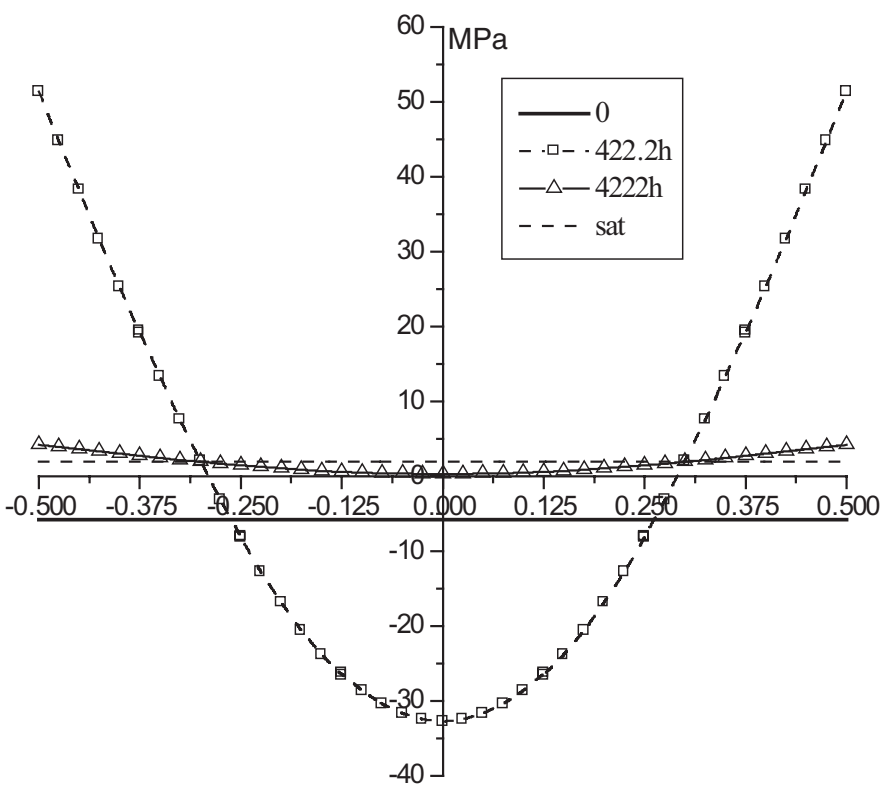

Figure 4. Residual transverse stress $\sigma_{y}^{r}$ through the thickness of a graphite/epoxy $[+10$, $-10]_{2 s}$ during desorption. 
A. TounsI ET AL.

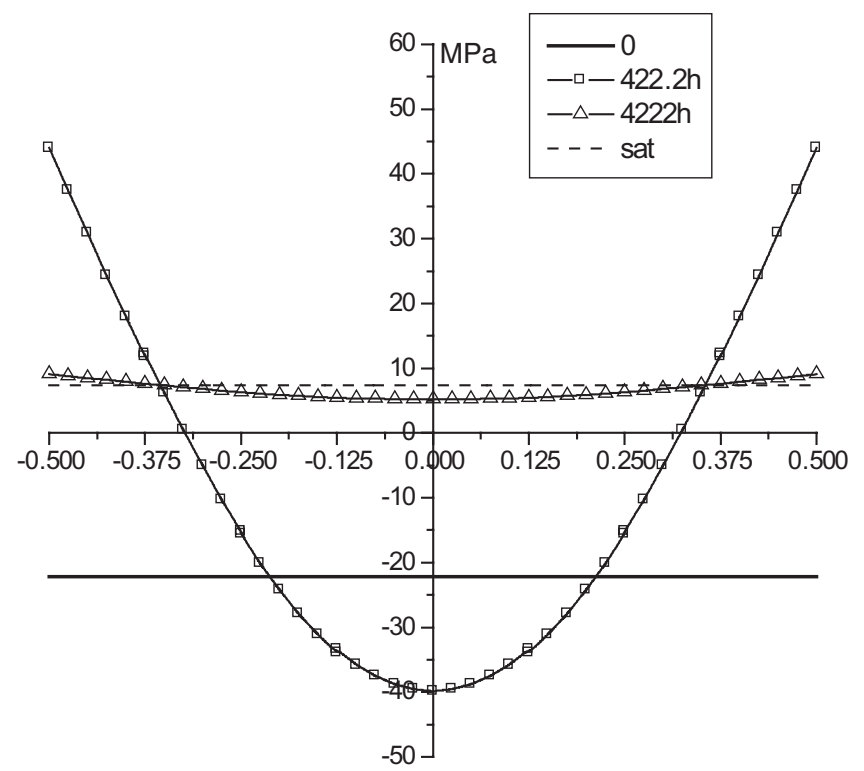

Figure 5. Residual transverse stress $\sigma_{y}^{r}$ through the thickness of a graphite/epoxy $[+20$, $-20]_{2 S}$ during desorption.

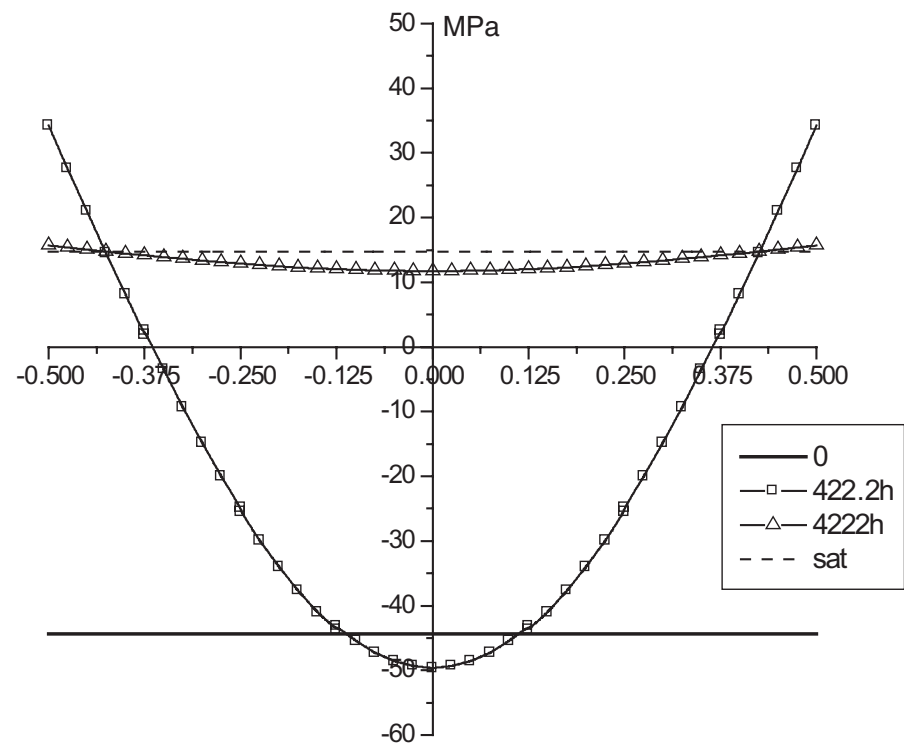

Figure 6. Residual transverse stress $\sigma_{y}^{r}$ through the thickness of a graphite/epoxy $[+30$, $-30]_{2 s}$ during desorption. 


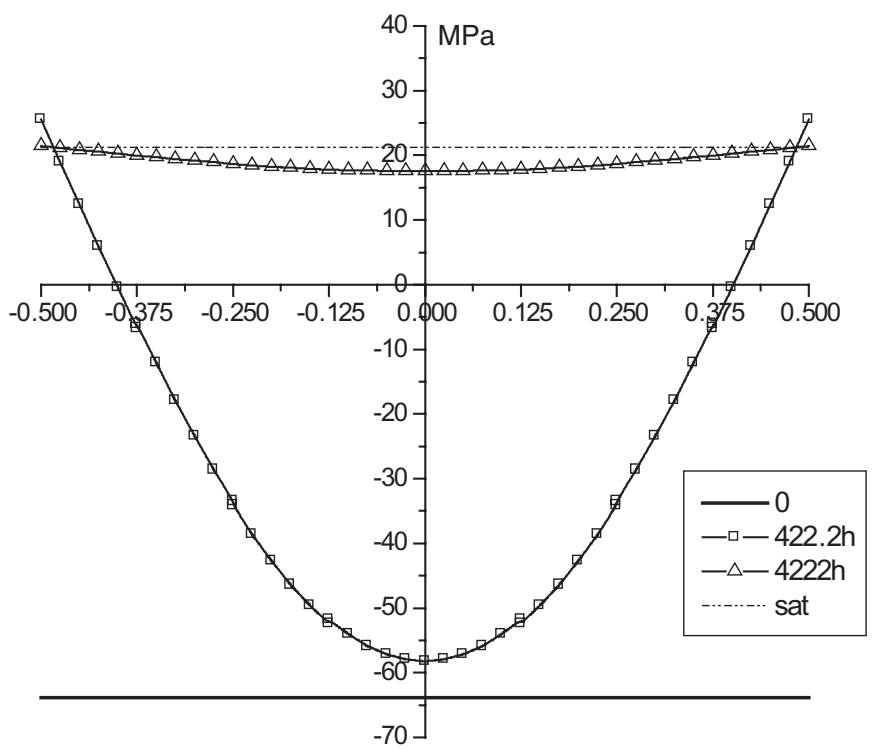

Figure 7. Residual transverse stress $\sigma_{y}^{r}$ through the thickness of a graphite/epoxy $[+45$, $-45]_{2 S}$ during desorption.

respectively. The magnitude of these stress peaks is maximum at the beginning of the desorption procedure. However, this magnitude decreases as $\theta$ increases and becomes approximately zero for $\theta=45^{\circ}$. In the latter case, the transverse stress distribution regularly increases from the initial to the final one. In all cases, the maximum tensile transverse stress cannot be neglected compared to the transverse tensile strength $Y$. However, as is mentioned in the case of unidirectional laminates, this calculation does not take into account the decrease of the moduli with the moisture concentration, which is likely to cause a stress relaxation.

\section{Influence of Anisotropy on the Transversal Transient Hygroscopic Stresses during Desorption Phase}

In the following investigations, the calculation of transient residual stresses within laminated plate is carried out at $t=422.2 \mathrm{~h}$.

Some configurations of materials were analyzed aiming to study the influence of anisotropy on the hygrothermal behavior of laminated composite plates during moisture desorption. Because of the importance of transverse residual stresses comparatively to both longitudinal and shear residual stresses, we have studied only the effect of the in-plane degree of anisotropy on the variation of such stresses. In fact, as is shown in 
"Transient Hygroscopic Stresses in the Unidirectional Laminate" and "Transient Hygroscopic Stresses in angle-ply $[+\theta,-\theta]_{2 S}$ Laminates" during moisture desorption the transverse residual stress $\sigma_{y}^{r}$ reaches considerable values compared to both the transverse tensile and compressive strengths $Y$ and $Y^{\prime}$. Also, in the case of moisture absorption, $\sigma_{y}^{r}$ is more important than $\sigma_{x}^{r}$ and $\sigma_{S}^{r}[1,4,5]$.

In Figures 8 and 9, the transversal hygroscopic stresses $\sigma_{y g}^{r}$ and the residual thermal stresses $\sigma_{y t}^{r}$ are plotted for laminates having different in-plane degree of anisotropy $\varepsilon_{A}$.

Figure 8 shows that for laminates with lower in-plane degree of anisotropy, the transverse transient stress $\sigma_{y g}^{r}$ decreases with the decrease in the in-plane degree of anisotropy $\varepsilon_{A}$. The state of this stress in the external plies is oriented towards a compressive state for laminates with lower degree of anisotropy and towards a tensile state for those having a higher degree of anisotropy; in contrast with the internal plies where they are in compressive state for all laminates considered here. However, we note that for laminates having a lower degree of anisotropy, the internal plies become in a more compressive state. On the other hand, the thermal residual stress $\sigma_{y t}^{r}$ (Figure 9) increases as soon as the in-plane degree of anisotropy $\varepsilon_{A}$ decreases. All plies of laminates are in

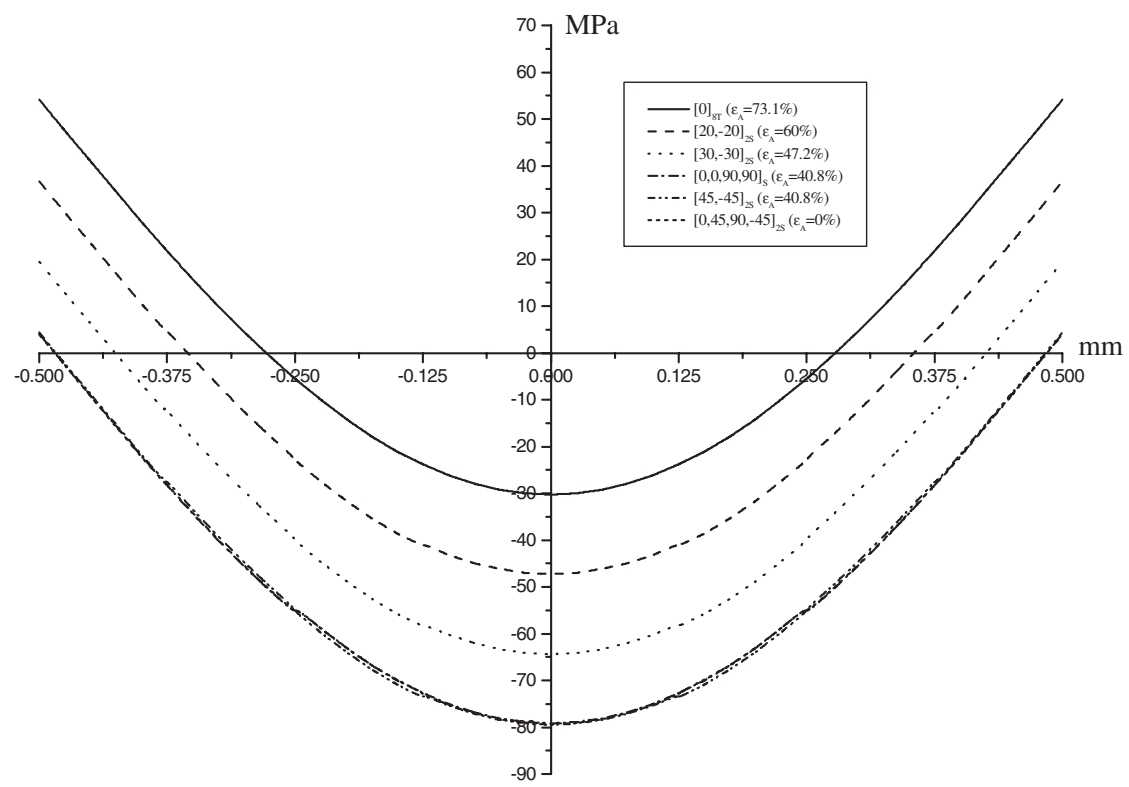

Figure 8. Influence of the degree of anisotropy $\varepsilon_{A}$ on the transversal hygroscopic $\sigma_{y g}^{r}$ through the thickness of the plate during moisture desorption $(t=422.2 \mathrm{~h})$. 


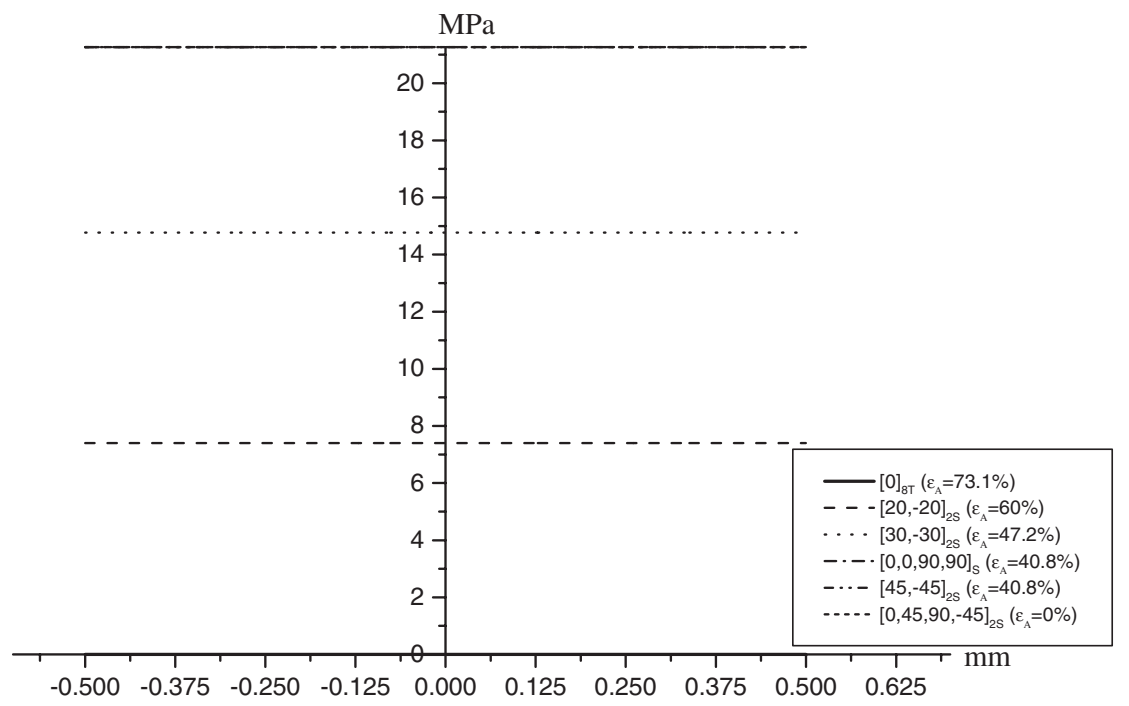

Figure 9. Influence of the degree of anisotropy $\varepsilon_{A}$ on the transverse residual thermal $\sigma_{y t}^{r}$ through the thickness of the plate during moisture desorption $(t=422.2 \mathrm{~h})$.

tensile state for this case, the same remark is observed in the case of moisture absorption [4,5]. As a result, the reduction of the in-plane degree of anisotropy favors the transversals hygroscopic stresses to be in compressive state, in contrast with the thermal stresses where they become in tensile state. This explains in a clear way the partial compensation of these stresses which are originally different.

Secondly, laminates with different lay-ups and the identical in-plane degree of anisotropy were investigated to see if they will lead to identical residual stresses. As is discussed in [5] in the case of moisture absorption: the values of the transverses residual stresses in laminates having the same inplane degree of anisotropy are nearly identical. The same remark is observed in the present case (moisture desorption). In fact, Figure 10 shows that for the three analyzed laminates which have almost a zero in-plane degree of anisotropy: $[0 / 45 / 90 /-45]_{2 \mathrm{~S}}\left(\varepsilon_{A}=0 \%\right),[60 /-60 / 0 /-60 / 0 / 60 / 0 / 60 /-60]$ $\left(\varepsilon_{A}=0 \%\right)$ and $[0 / 59 / 107 / 129 / 50 / 130 / 178 / 21 / 69 / 128]\left(\varepsilon_{A}=0.2 \%\right)$; the distribution of the transverse hygroscopic stresses $\sigma_{y g}^{r}$ is almost identical. Figure 11 shows also an analogous behavior for the three other considered laminates which have almost an identical in-plane degree of anisotropy: [45/ $-45]_{2 \mathrm{~S}}\left(\varepsilon_{A}=40.8 \%\right),[0 / 0 / 90 / 90]_{\mathrm{S}}\left(\varepsilon_{A}=40.8 \%\right)$ and $[0 / 90 / 0 / 90 / 0 / 90 / 0 / 90 / 0]$ $\left(\varepsilon_{A}=41.8 \%\right)$. This latter investigation confirms also this important result. Finally, other studies are scheduled to detail this point as is presented in $[4,5]$. 
A. Tounsi ET AL.

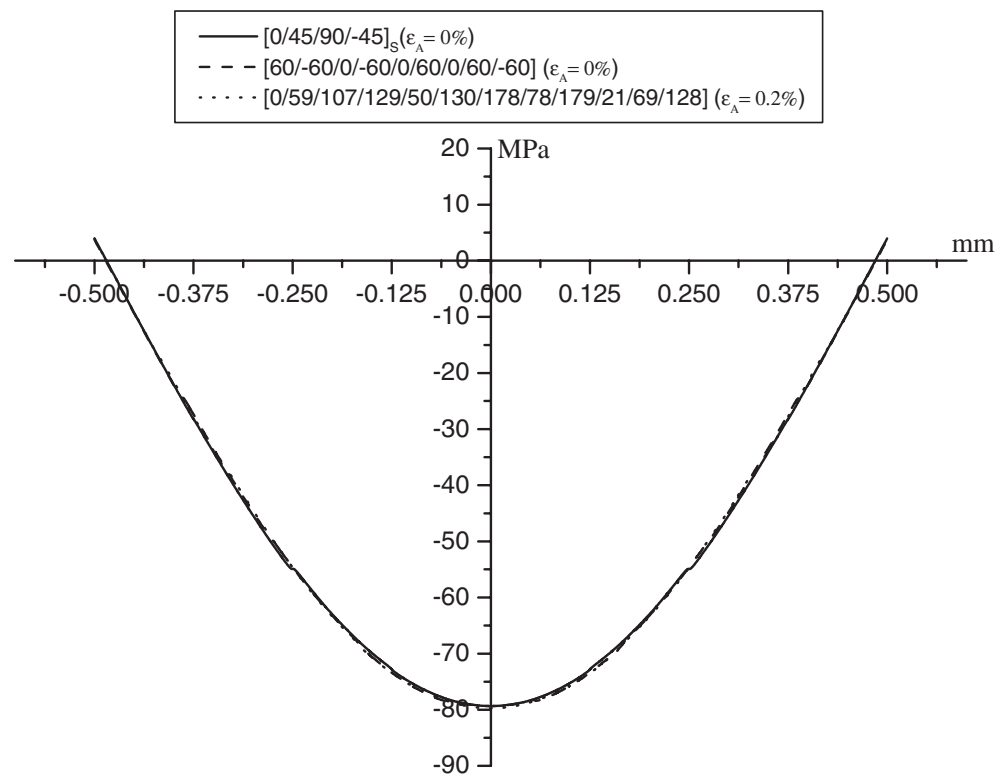

Figure 10. Residual transverse stress $\sigma_{y}^{r}$ through the thickness of the quasi-isotropic laminates.

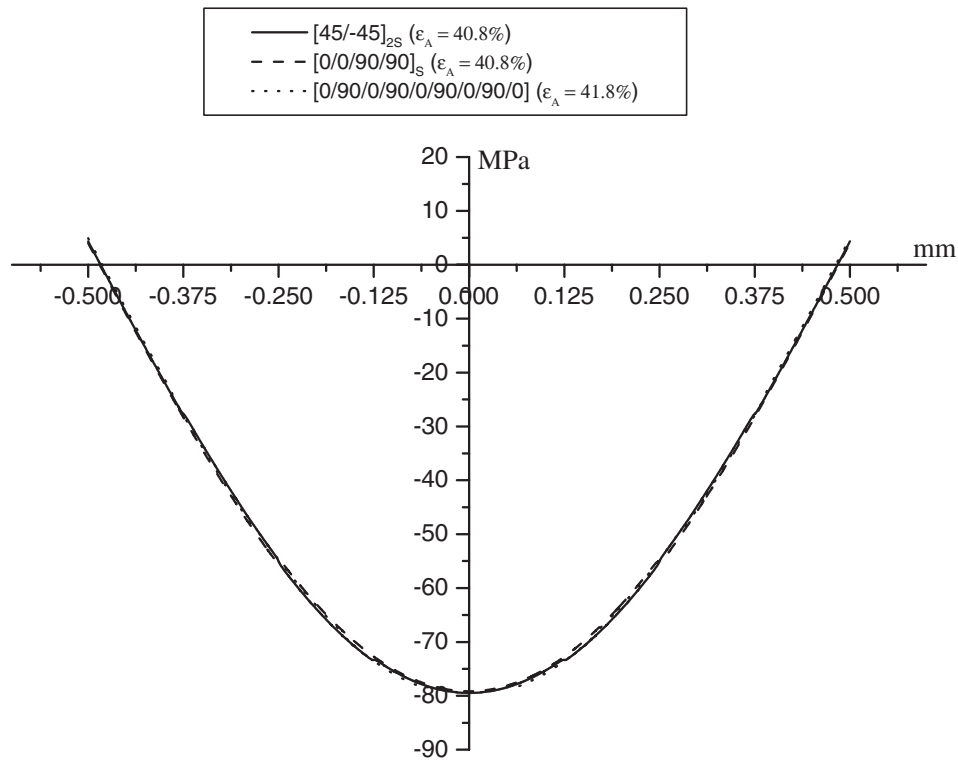

Figure 11. Residual transverse stress $\sigma_{y}^{r}$ through the thickness of laminates having almost the same in-plane degree of anisotropy. 


\section{CONCLUSION}

In this work the transient hygroscopic stresses induced by the symmetric environmental conditions during moisture desorption in laminated composite plates were studied. For that, a straightforward and quick analytical method is presented to determine such stresses in the polymer matrix composite plate. Some stacking sequences, such as those of unidirectional and angle-ply laminates, exhibit non-negligible transverse stress peaks compared to transverse strength.

The second part of this paper, is devoted to the analysis of the effect of anisotropy on the variation of the transversal non-mechanical residual stresses across laminated composite plates. It was shown that the reduction of the in-plane degree of anisotropy favors the transversals hygroscopic stresses to be in compressive state, in contrast with the thermal stresses where they become in tensile state. Surprisingly, our investigation shows that: for the considered laminates and which have the same in-plane degree of anisotropy and different lay-up, we will have an identical distribution of transversal hygroscopic stresses through the thickness of these laminates.

Finally, the present simplified approach could be extended to advanced mechanical behavior including the influence of moisture and creep on the stiffnesses.

\section{NOMENCLATURE}

$D=$ diffusivity

$C=$ moisture concentration

$N=$ number of the ply, or number of the subply

$N_{a}^{n}=$ the in-plane stress resultant components

$M_{\alpha}^{n}=$ moment components

$X=$ longitudinal tensile strength

$X^{\prime}=$ longitudinal compressive strength

$Y=$ transverse tensile strength

$Y^{\prime}=$ transverse compressive strength

$S=$ longitudinal shear strength

$h=$ thickness of plate

$z=$ axis transverse to the plane of laminate; or thickness direction of a laminate

$h_{k}=$ thickness of ply; or thickness of subply (when we want to calculate the transient hygroscopic stresses inside the ply)

$\left\{k^{n}\right\}=$ nonmechanical curvature

$\Delta T=$ room (or operating) temperature - cure temperature 


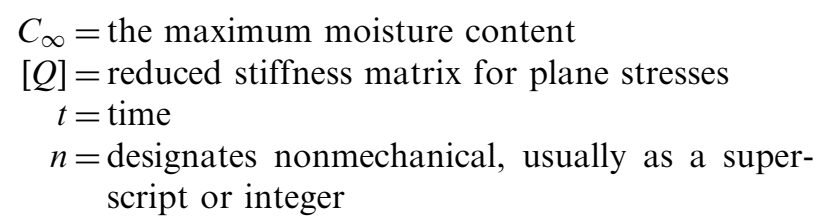

$p_{n}, q_{n}, p_{\alpha n}, Q_{\alpha n}, p_{\beta n}, Q_{\beta n}=$ quantities used only to simplify the mathematical equations

$A_{\theta}, B_{\theta}, C_{\theta}, D_{\theta}, E_{\theta}, F_{\theta}=$ quantities used only to simplify the mathematical equations

$\begin{aligned} \mathrm{e}_{\mathrm{i}}{ }^{k} ;(i=x, y, s) & =\text { the free expansions at the point } z_{k} \\ \alpha_{i} ;(i=x, y, s) & =\text { thermal expansion coefficients } \\ \beta_{i} ;(i=x, y, s) & =\text { moisture expansion coefficients } \\ \lambda_{k} & =\text { the moisture concentration at the point } z_{k}\end{aligned}$ $\left(\lambda_{k}=C\left(z_{k}, t\right)\right)$

$\sigma_{i}^{n}(i=x, y, s)=$ nonmechanical stresses component in the ply axis

$\sigma_{i}^{r}=$ residual stress components in the ply axis

$\sigma_{i g}^{r}=$ transient hygroscopic stresses

$\sigma_{i t}^{r}=$ thermal residual stresses

$\varepsilon_{i}^{r}=$ residual strain components in the ply axis

$\sigma_{\alpha}^{n}(\alpha=1,2,6)=$ nonmechanical stresses component in the laminates axis

$\psi_{k}, \xi_{k}, \rho_{k}=$ quantities used only to simplify the mathematical equations

$\theta=$ ply angle

$[\alpha]=$ the in-plane sub-matrix of the compliance components of laminate

$[\beta]=$ the in-plane/flexure coupling sub-matrix of the compliance matrix of laminate

$[\beta]^{\mathrm{t}}=$ transposed $[\beta]$

$[\delta]=$ flexural sub-matrix of the compliance matrix of laminate

$\left\{\varepsilon^{0 n}\right\}=$ nonmechanical in-plane strain components

\section{REFERENCES}

1. Tounsi, A. and Adda-Bedia, E.A. (2003). Simplified Method for Prediction of Transient Hygroscopic Stresses in Polymer Matrix Composites with Symmetric Environmental Conditions, International Journal of Applied Composite Materials, 10(1), 1-18.

2. Benkeddad, A., Grediac, M. and Vautrin, A. (1996). Computation of Transient Hygroscopic Stresses in Laminated Composite Plates, Journal of Composites Science and Technology, 56: 869-876. 
3. Benkeddad, A., Grediac, M. and Vautrin, A. (1995). On the Transient Hygroscopic Stresses in Laminated Composite Plates, International Journal of Composite Structures, 30(2), 201-205.

4. Tounsi, A., Adda-Bedia, E.A., Verchery, G. (2002). Influence of Anisotropy on the Transient Hygroscopic Stresses in Polymer Matrix Composites with Cyclic Environmental Conditions, International Journal of Composite Structures, 55(4), 393-405.

5. Tounsi, A. and Adda Bedia, E.A. Some Observations on the Evolution of Transversal Hygroscopic Stresses in Laminated Composites Plates: Effect of Anisotropy, International Journal of Composite Structures, 59(4), 445-454, 203.

6. Tounsi, A., Adda-Bedia, E.A., Sereir, Z. and Verchery, G. (2000). Analyse de l'effet d'anisotropie et des conditions d'environnement cycliques sur les variations des contraintes hygroscopiques transitoires à l'intérieur des plaques composites stratifiées, Communication au $12^{\text {ème }}$ journées nationales sur les composites, pp. 969-978, JNC12, 15, 16 et 17 Novembre 2000 (in French), France.

7. Adda-Bedia, E.A., Tounsi, A. and Sereir, Z. (2001). A Quantitative Study on the Influence of Anisotropy on the Hygrothermal Behaviour of the Laminated Composite Plates, Eighth International Conference on Composite Engineering ICCE8, p. 71, August 5-11, Tenerife, Spain.

8. Cheikh Saad Bouh, A.B. and Verchery, G. (1992). Influence of Mechanical Anisotropy on the Bending of Laminates, In: Proceedings of the Second International Symposium on Textile Composites in Building Construction (TCIBC 92) Part 2, pp. 149-162, June 23-25, Lyon, French.

9. Cheikh Saad Bouh, A.B., Aivazzadeh, S., El Sheikh, M.S. and Verchery, G. (1991). A Quantitative Study of the Influence of Anisotropy on the Bending Deformation of Laminates, 8th International Conference on Composite Materials, pp. 1-12, Honolulu (USA), Section 26-J.

10. Vincenti, A., Verchery, G. and Vannucci, P. (2001). Anisotropy and Symmetry for Elastic Properties of Laminates Reinforced by Balanced Fabrics, Composites Part A, 32(10): 1525-1532.

11. Vannucci, P. and Verchery, G. (2001). Stiffness Design of Laminates using the Polar Method, International Journal of Solids and Structures, 38: 9281-9294.

12. Verchery, G. (1999). Designing with Anisotropy - Part 1: Methods and General Results for Laminates, Keynote Lecture. 12th International Conference on Composite Materials (ICCM-12), Paris.

13. Vannucci, P. and Verchery, G. (1999). Designing with Anisotropy - Part 2 : Laminates without Membrane-flexure Coupling, 12th International Conference on Composite Materials (ICCM-12), Paris.

14. Vannucci, P., Cong, X.J. and Verchery, G. (1999). Designing with Anisotropy - Part 3: Quasi-Homogeneous Anisotropic Laminates, 12th International Conference on Composite Materials (ICCM-12), Paris.

15. Vincenti, A., Verchery, G. and Vannucci, P. (2000). Découplage et quasi-homogénéité pour les stratifiés renforcés par tissus équilibrés, pp. 265-274, JNC12, (in French), France.

16. Hahn, H.T. and Kim, R.Y. (1978). Swelling of Composite Laminates, Advanced Composite Materials - Environmental Effects, ASTM STP 658, pp. 98-120.

17. Springer, G. (1981). Environmental Effects on Composite Materials, Technomic Publishing, Westport.

18. Adda-Bedia, E.A., Han, W.S. and Verchery, G. (2000). An Asymptotic Characterisation of the Moisture Diffusion in Polymer Matrix Composites with Cyclic Environmental Conditions, International Journal of Composite Structures, 49(3): 269-274.

19. Adda-Bedia, E.A., Han, W.S. and Verchery, G. (1998). Simplifed Methods for Prediction Diffusion in Polymer Matrix Composites with Cyclic Environmental Conditions, International Journal of Polymer \& Polymer Composites, 6 (14), 189-203. 
20. Adda-Bedia, E.A., Han, W.S. and Verchery, G. (1993). Edge Effects in Resin Matrix Composites with Cyclic Environmental Conditions, In: Miravet, A. (ed.), Proceeding of the 9th International Conference on Composite Materials, (ICCM-9), 5, 617-624.

21. Adda-Bedia, E.A., Han, W.S. and Verchery, G. (1992). Moisture Diffusion in Polymer Matrix Composites with Cyclic Environmental Conditions, Textile Composite in Building Construction (TCIBC 92), In: Proceedings of the International Symposium, Part 2, pp. 127-138, Lyon, France, (Pluralis, Paris).

22. Adda-Bedia, E.A. (1994). Méthode de Résolution Pour la Diffusion de l'Humidité sous les Conditions d'Environnement Cycliques, Doctoral Dissertation (in French), Université Claude Bernard Lyon I, Lyon, France.

23. Tsai S.W. (1988). Composite Design, 4th edn, Think Composites, Dayton.

24. Verchery, G. (1979). Les invariants des tenseurs d'ordre quatre du type de l'élasticité, In: Proceedings of the Euromech Colloquium 115, pp. 93-104, Villards-de-Lans. Editions du CNRS in 1982, Paris (in French).

25. Grédiac, M., Vautrin, A. and Verchery, G. (1993). A General Method for Data Averaging of Anisotropic Elastic Constant, International Journal of Appl. Mech., 60: 614-618.

26. Kandil, N. and Verchery, G. (1990). Design of Stacking Sequences of Laminated Plates for Thermoelastic Effects, In: De Wilde, W.P. and Blain, W.R. (eds), Proceedings of the 2nd International Conference on Computer Aided Design in Composite Materials Technology, pp. 565-576, Brussels, Computational Mechanics Publications, Southampton.

27. Verchery, G. and Vong, T.S. (1986). Une méthode d'aide graphique à la conception des sequences d'empilement dans les stratifiés, comptes rendus de JNC 5, pp. 267-280 (in French).

28. Jones, R.M. (1975). Mechanics of Composite Materials, Taylor and Francis, USA.

29. Grédiac, M. (1996). On the Direct Determination of Invariant Parameters Governing Anisotropic Plate Bending Problem, International Journal of Solids and Structure, 33(27): 3969-3982.

30. Vergnaud, J.M. (1992). Drying of Polymeric and Solid Materials: Modelling and Industrial Applications, Springer-Verlag, London.

31. Surrel, Y. and Vautrin, A. (1989). On the Modelling of the Plastic Response of FRP Under Monotonic Loading, International Journal of Composite Materials, 23: 232-250. 